\title{
ANÁLISE DE SISTEMAS A EVENTOS DISCRETOS VIA MODELOS MODULARES: APLICAÇÃO A SISTEMAS DE SERVIÇO COM ABANDONO DE CLIENTE.
}

\author{
Lisley Mara Guimarães de Souza* Carlos Andrey Maia* \\ * Programa de Pós-Graduação em Engenharia Elétrica - Universidade \\ Federal de Minas Gerais (UFMG) - Av. Antônio Carlos 6627, \\ 31270-901, Belo Horizonte, MG, Brasil (e-mail: lisleysouza@ufmg.br ; \\ maia@cpdee.ufmg.br).
}

\begin{abstract}
The work of constructing a model which adequately represents the functioning of a system is of great complexity because large systems with diverse functionalities are generally analyzed. Therefore, techniques with a modular character become an attractive alternative for the modeling of systems. This article proposes a methodology that approaches the modeling through the theory of Systems to Discrete Events, the implementation and the analysis of the results, in order to model and analyze client-server systems with the presence of impatient clients. Through a modular way by means of stochastic automata and through the properties of the Markov chain and queuing theory it is possible to analyze the proposed system. For the comparison question, the same modeling was used to analyze the performance of the system by the analytical method and simulation.

Resumo: A tarefa de construir um modelo, que represente adequadamente o funcionamento de um sistema, é de grande complexidade por geralmente serem analisados sistemas grandes e com diversas funcionalidades. Por isso, técnicas com um caráter modular tornam-se uma alternativa atraente para a modelagem de sistemas. Este artigo propõe uma metodologia que aborda a modelagem através da teoria de Sistemas a Eventos Discretos, a implementação e a análise dos resultados, para modelar e analisar sistemas do tipo cliente-servidor com presença de clientes impacientes, de forma modular por meio de autômatos estocásticos e por meio das propriedades da cadeia de Markov e da teoria de filas analisar o sistema proposto. Para quesito de comparação, foi utilizada a mesma modelagem para a análise de desempenho do sistema via método analítico e por simulação.
\end{abstract}

Keywords: Automata; Discrete Event Systems; Impatient Customers; Performance evaluation; Queuing Theory; System modeling.

Palavras-chaves: Autômatos; Avaliação de desempenho; Clientes impacientes; Modelagem de sistemas; Sistemas a Eventos Discretos; Teoria de filas.

\section{INTRODUÇÃO}

A análise de desempenho de sistemas é de grande importância para verificar possíveis otimizações, ajustes e detectar possíveis falhas. Para uma análise adequada é necessária a construção de um modelo que represente todas as partes do sistema que será analisado.

Neste artigo são analisados sistemas do tipo cliente servidor, que são caracterizados como sistemas em que há presença de clientes em fila aguardando atendimento. Como existe a espera dos clientes pelo serviço, há também

\footnotetext{
* Este trabalho tem como parceiros e apoiadores o CNPQ, a FAPEMIG e a CAPES, em conjunto com o Programa de Pós Graduação de Engenharia Elétrica da Universidade Federal de Minas Gerais (UFMG).
}

o abandono do mesmo, e um dos desafios para modelagem desse tipo de sistemas é considerar a paciência dos clientes.

O estudo da paciência dos clientes é uma área que está em desenvolvimento e em busca de melhorias. Na literatura os trabalhos mais voltados à impaciência de clientes são direcionados aos call centers, podem-se citar os artigos: ROUBOS (2013), WHITT (2004), YUE (2014), ALSEEDY (2009), ABOU-EL-ATA (1992), entre outros.

Então, para uma melhor eficiência é necessário estimar a impaciência dos clientes (SOUZA, 2019). Para isso, foi elaborado uma metodologia a fim de analisar o desempenho de sistemas do tipo cliente servidor, utilizando uma modelagem modular por meio dos autômatos estocásticos, utilizando métodos analítico e por simulação aplicando as propriedades da cadeia de Markov e da teoria de filas. 


\section{SISTEMAS A EVENTOS DISCRETOS}

Os sistemas que são considerados neste artigo são os Sistemas a Eventos Discretos (SED). Os SED captam a ocorrência externa por meio de estímulos denominados eventos, podendo esses eventos ser o início ou término de uma tarefa; as ocorrências desses estímulos causam uma alteração interna no sistema, não dependendo da passagem do tempo para evoluir como acontece nos sistemas contínuos, (CASSANDRAS, 2008).

\subsection{Autômatos}

Os autômatos são uma ferramenta que podem representar o comportamento de um SED por meio de uma linguagem com regras bem definidas (CASSANDRAS, 2008). Podendo ser classificado como determinístico ou estocástico, temporizado ou não temporizado, finito ou infinito.

O autômato é utilizado para representar a dinâmica de um sistema, em que os eventos são temporizados. Então, é necessária uma estrutura de temporização que defina uma sequência de tempo de vida para os eventos, podendo o tempo ser determinístico ou estocástico. Para cada estado são verificadas quais transições são factíveis, bem como os momentos de ocorrência (RIBEIRO, 2018). Uma estrutura de relógio $(\mathrm{V})$ associado a um evento $e$ pode ser definido como um conjunto (1):

$$
V=\left\{v_{i}: i \in E\right\}
$$

em que $v_{i}$ corresponde uma sequência de vida para cada evento $i$ pertencente ao conjunto de eventos. Geralmente, os eventos ocorrem de forma estocástica, ou seja, existe uma incerteza em relação ao próximo evento. Segundo CASSANDRAS (2008), um autômato temporizado estocástico é composto por uma estrutura de relógio estocástica, em que para cada evento aleatório, é relacionada uma função de distribuição que caracteriza o comportamento do mesmo. CASSANDRAS (2008) define um autômato temporizado estocástico por uma sêxtupla $\left(X, E, \Gamma, p, p_{0}, H\right)$, em que:

- $X$ é o conjunto finito de estados;

- E é o conjunto finito de eventos associados ao autômato;

- $\Gamma: X \rightarrow 2^{E}$ é a função de eventos factíveis. $\Gamma(x)$ representa todos os eventos ativos dado que o autômato se encontra no estado $x$;

- $p\left(x^{\prime} ; x, e\right)$ é a probabilidade de transição do estado $x$ para $x^{\prime}$, dada a ocorrência do evento $e$ sendo que $p\left(x^{\prime} ; x, e\right)=0 \forall e \notin \Gamma(x)$;

- $p_{0}$ é a probabilidade do estado inicial do sistema ser $x$;

- H é uma estrutura de relógio estocástica.

Modelagem Modular Uma das formas que pode ser utilizada para modelar sistemas grandes e complexos é por meio da modelagem modular. Para isso, são necessários vários autômatos para representar a dinâmica do sistema como um todo, então ao construir o sistema por parte são necessárias operações que modifiquem o seu diagrama de transição. CASSANDRAS (2008) define duas principais operações em autômatos: produto, indicado por $\times$, e a composição paralela, denotada por $\|$.
A operação por produto é mais restritiva, pois as transições dos autômatos devem estar sempre sincronizadas, um evento só ocorre se, e somente se, ocorrer em todos os autômatos (CASSANDRAS, 2008). Então é mais usual utilizar a composição paralela para modelar sistemas modulares compostos por vários autômatos que interagem, com eventos privados e eventos em comum. Na composição paralela, quando o evento é comum, ele só pode ser executado se todos os autômatos puderem executá-lo simultaneamente, fazendo assim com que os autômatos sejam sincronizados nos eventos que são comuns. Já os eventos privados podem ser disparados sempre que possíveis, sem nenhuma restrição (CASSANDRAS, 2008).

\section{CADEIA DE MARKOV}

Em janeiro de 1913, o matemático russo A. Markov fundou um novo ramo da teoria da probabilidade, conhecido como cadeia de Markov (HAYES, 2013). WANG (2011) define a cadeia de Markov como um método de previsão de probabilidade e técnica para prever os próximos estados do sistema, estudando apenas o estado atual e sua tendência de mudança no sistema. Por isso, a cadeia de Markov é utilizada para descrever e analisar propriedades de sistemas dinâmicos, cuja principal característica é determinar a probabilidade do sistema estar em qualquer estado em qualquer instante de tempo (CASSANDRAS, 2008).

\subsection{Autômato estocástico visto como uma cadeia de Markov}

Ao modelar um sistema de forma modular com vários autômatos estocásticos compostos por uma distribuição exponencial, é possível obter o comportamento do sistema através da composição paralela, e também obter facilmente a cadeia de Markov que representa esse sistema, pois a transição entre estados é também pode ser escrita em função de taxas de transferência CASSANDRAS (2008).

Além do conjunto de estados $X$, uma cadeia de Markov que representa um autômato estocástico é composta por uma matriz de taxas de transição $M_{q}$ (RIBEIRO, 2018), representada abaixo:

$$
M_{q}=\left(\begin{array}{ccccc}
q_{00} & q_{01} & q_{02} & \cdots & q_{0 L} \\
q_{10} & \cdots & \cdots & \cdots & q_{1 L} \\
\vdots & \vdots & \vdots & \vdots & \vdots \\
q_{L 0} & \cdots & \cdots & \cdots & q_{L L}
\end{array}\right)
$$

SOUZA (2018) explica que L representa o número de estados e $q_{i j}$, a taxa de transição do estado i para o estado j. A definição de $q_{i j}$ é dada por $\lambda_{a}$ se $i \neq j$, em que $\lambda_{\alpha}$ é a taxa de ocorrência de um evento $\alpha$, ou $-\sum_{\substack{i \neq j \\ j=0}}^{L} q_{i j}$ se $i=j$. Assim, pode-se determinar a probabilidade do sistema estar em um estado em qualquer instante de tempo, como:

$$
\pi M_{q}=0
$$

onde $\pi$ representa um vetor composto por cada $\pi_{i} \forall x_{i} \in X$. Assim, a probabilidade estacionária de cada estado pode ser obtida resolvendo um sistema de equações lineares RIBEIRO (2018). Para mais detalhes de como derivar a cadeia de Markov do autômato estocástico ver: CASSANDRAS (2008); RIBEIRO (2018); SOUZA (2019). 


\section{TEORIA DE FILAS}

A teoria de filas foi desenvolvida com o objetivo de fornecer modelos para prever o comportamento de sistemas de fila que fornecem serviços para demandas surgidas aleatoriamente, (GROSS et al., 2008). Um sistema de fila pode ser representado como clientes que chegam para o serviço, clientes aguardando pelo serviço, que se não for imediato e tiverem que aguardar pelo serviço formam uma fila, e o cliente deixando o sistema após ser atendido (SOUZA, 2019). O termo cliente é usado em um sentido geral e não implica necessariamente um cliente humano.

GROSS et al. (2008) cita seis características básicas dos processos de teoria das filas, em que existe uma notação consolidada na literatura através de uma série de símbolos e barras, que descreve resumidamente o modelo do sistema (GROSS et al., 2008), essa notação é a seguinte:

$$
A / B / m / K / Z
$$

Em que:

- $A$ é a distribuição do tempo entre as chegadas;

- $B$ é a distribuição do tempo de serviço;

- $m$ é a quantidade de servidores do sistema;

- $K$ é a capacidade do tamanho da fila;

- $Z$ é a disciplina da fila.

\subsection{Lei de Little}

No início dos anos 1960, John Little relacionou os tamanhos médios do sistema em estado estacionário aos tempos médios de espera do cliente, que é uma das relações mais poderosas na teoria das filas, conhecida como lei de Little (GROSS et al., 2008). A Figura 1 ilustra a relação da lei de Little.

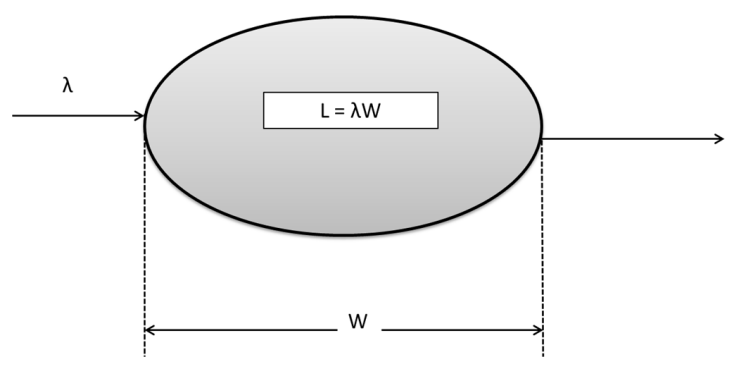

Figura 1. Lei de Little

Considerando que $\lambda$ é a taxa de chegada dos clientes, $W_{q}$ e $W$ são o tempo médio de espera na fila e o tempo médio de espera no sistema, as fórmulas de Little são:

$$
L=\lambda W \quad e \quad L_{q}=\lambda W_{q},
$$

em que $L$ e $L_{q}$ são o número médio de clientes no sistema e número médio de clientes esperados na fila, respectivamente. Um resultado interessante que pode ser derivado das fórmulas de Little em relação ao tempo de espera é:

$$
L-L_{q}=\lambda\left(W-W_{q}\right)=\lambda\left(\frac{1}{\mu}\right)=\left(\frac{\lambda}{\mu}\right)
$$

\section{SISTEMAS DO TIPO CLIENTE - SERVIDOR COM ABANDONO}

Uma característica importante na modelagem de sistemas que possuem fila é a presença de clientes impacientes. GROSS et al. (2008) explica que pode-se dividir a paciência em duas principais formas: balking e reneging.

De acordo com Wang et al. (2010), o balking é quando o cliente decide de acordo com o tamanho da fila. Muitas vezes os clientes sentem-se desencorajados quando a fila é longa e não desejam esperar por muito tempo.

Já o abandono dos clientes através do reneging, os clientes que tendem a ser impacientes podem entrar na fila para ver quanto tempo a sua espera pode estender, permanecendo o tempo todo ou abandonando se a estimativa de sua espera total for maior que sua tolerância (GROSS et al., 2008).

ROUBOS (2013) propõe no seu artigo modelar a paciência dos clientes através da distribuição hiperexponencial, que é uma mistura de duas distribuições exponenciais tais que com probabilidade $p$ com taxa $\gamma_{1}$ e com probabilidade $(1-p)$ com taxa $\gamma_{2}$.

Um sistema do tipo cliente - servidor com abandono pode ser visto na Figura 2, em que existe a chegada de dois tipos de cliente, por meio dos eventos $a_{1}$ e $a_{2}$, a admissão do cliente pelo servidor via eventos $c_{1}$ e $c_{2}$, o evento $d$ representa a saída dos clientes do sistema após o atendimento e também o abandono dos clientes através dos eventos $b_{1}$ e $b_{2}$ (SOUZA, 2019).

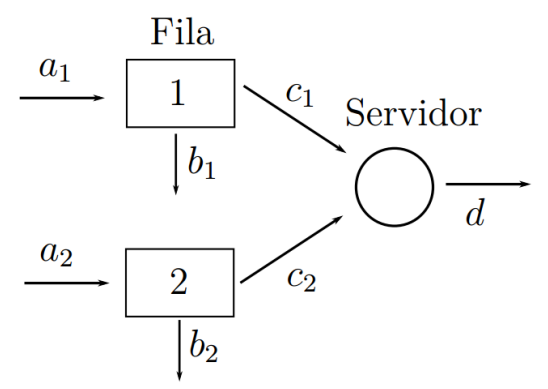

Figura 2. Sistema de clientes - servidor com abandono

\section{METODOLOGIA}

Para analisar o desempenho de um sistema, primeiro é necessário conhecer cada parte do processo e quais são os parâmetros de interesse que se deseja obter e adquirir melhorias. Definido os parâmetros de interesse do sistema cliente-servidor, primeiramente foi elaborado um modelo para representar o sistema através da modelagem modular via autômatos estocásticos e depois utilizando as propriedades da cadeia de Markov e teoria de filas foi realizada a análise de desempenho do sistema de filas via método analítico e por simulação.

Depois de construir do modelo, o segundo passo foi implementar o modelo modular via autômatos estocásticos na biblioteca UltraDES, uma biblioteca que implementa estruturas para representar eventos, estados e autômatos (LACSED - UFMG, 2016). Em seguida, para o método analítico, foi realizada a operação de composição paralela 
para a obtenção do modelo completo que representa a dinâmica do sistema como um todo, para assim obter a matriz $M_{q}$.

Obtendo a matriz de probabilidade de transição, foi elaborado um algoritmo para a resolução da matriz e análise dos parâmetros de interesse.

Para o método via simulação, não foi necessário realizar a composição paralela, apenas as matrizes de transição de cada autômato. Foi utilizada uma técnica padrão descrita no livro do CASSANDRAS (2008), conhecida como escalonamento de eventos, que consiste em simular a dinâmica do sistema e analisar o seu comportamento.

\subsection{Modelagem modular do problema por autômatos}

A paciência dos clientes foi modelada de acordo com a impaciência do tipo balking, em que os clientes observam o comprimento da fila e abandonam ou não de acordo com um comprimento " $N$ ". E para a impaciência do tipo reneging foi considerado que os clientes decidem esperar ou não, de acordo com o tempo de espera $T$ que segue uma distribuição exponencial com parâmetro $i$, em que $i$ é o número de clientes no sistema.

A Figura 3 mostra o comportamento da chegada do cliente do tipo 1, a chegada desse tipo de cliente é de acordo com uma probabilidade $p$ multiplicado pela taxa de chegada $\lambda$, representado pelo evento $a_{1}$. A fila tem capacidade $l$ e o decremento da fila se dá pela admissão do cliente pelo servidor, através do evento $c_{1}$ ou pelo abandono por meio do evento $b_{1}$, com taxa $i \gamma_{1}$, em que o $i$ representa o número de clientes na fila (SOUZA, 2019).

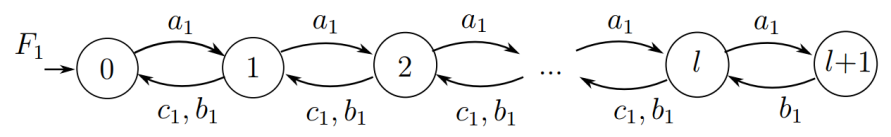

Figura 3. Cliente tipo 1

Assim como os clientes do tipo 1, SOUZA (2019) definiu que os clientes do tipo 2 chegam ao sistema mediante o evento $a_{2}$ com uma probabilidade de $(1-p)$ multiplicado pela taxa de chegada $\lambda$. A fila possui capacidade $n$ e o decremento da fila acontece com admissão do cliente pelo servidor através do evento $c_{2}$ ou pelo abandono por meio do evento $b_{2}$ por uma taxa $i \gamma_{2}$. A Figura 4 mostra o autômato da fila do cliente do tipo 2 .

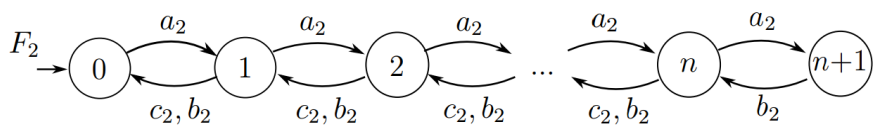

Figura 4. Cliente tipo 2

A diferença do comportamento das filas dos clientes do tipo 1 e 2 estão na taxa de ocorrência do abandono e, também na probabilidade de chegada de cada tipo de cliente, de acordo com o valor $p$ (SOUZA, 2019). Observando as Figuras 3 e 4, pode-se perceber que a partir dos estados $l$ e $n$ os clientes já não aguardam atendimento e abandonam o sistema com os eventos $b_{1}$ e $b_{2}$, seguindo a regra balking.
Também é possível verificar a regra reneging em que as taxas de abandono $\gamma$ aumentam de acordo com a quantidade de clientes no sistema.

A Figura 5 ilustra o comportamento dos servidores, que através dos eventos $c_{1}$ ou $c_{2}$ admitem um cliente para atendimento, e finalizam o serviço com o evento $d$. O tempo de vida do evento $d$ é calculado de acordo com a quantidade de clientes em atendimento com o limite máximo de $s$ servidores.

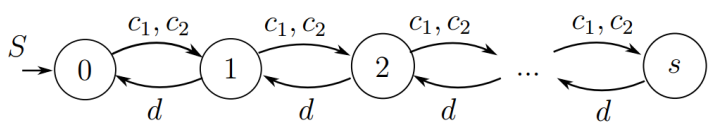

Figura 5. Servidores

A equação 5 representa o autômato geral obtido para o comportamento do sistema completo.

$$
G=F 1 \| F 2|| S
$$

Portanto, através dessa modelagem modular composta de três autômatos é possível realizar o estudo para a impaciência dos clientes em sistemas que possuem fila de espera e com $s$ servidores.

\subsection{Parâmetros de interesse}

Os parâmetros de interesse definidos para o sistema de fila foram a taxa de produção do sistema, também considerada como nível de serviço, que representa a quantidade de clientes que são atendidos de acordo com a taxa de chegada e tempo de atendimento. A probabilidade de sistema vazio, ou seja, a probabilidade do cliente chegar e não encontrar nenhum cliente no sistema. E o tempo médio de sistema, que é o tempo total que o cliente permanece no sistema, desde a sua chegada até sua saída.

Para isso foi utilizada as propriedades da cadeia de Markov e da teoria de fila, juntamente com a modelagem do sistema através da distribuição hiperexponencial.

Para o método analítico, como mostrado na equação 6, a taxa de produção ou nível de serviço do sistema foi calculada de acordo com a probabilidade dos servidores serem encontrados processando de $1,2, \ldots, m$ clientes. As probabilidades de cada estado são encontradas, através da cadeia de Markov, ao resolver a equação $\pi M_{q}=0$.

$$
\lambda_{p}=\pi_{x x 1} \times \lambda_{d}+\pi_{x x 2} \times 2 \lambda_{d}+\ldots+\pi_{x x m} \times m \lambda_{d} .
$$

Para o método de simulação a taxa de produção foi calculada através da quantidade de ocorrência dos eventos $d$, dividido pelo tempo total de simulação.

Para utilizar as equações da teoria de filas foi necessário encontrar a taxa de chegada efetiva $(\hat{\lambda})$ dos clientes no sistema, ou seja, a quantidade de clientes que realmente permanecem no sistema aguardando até o atendimento. De acordo com o teorema de Burke se uma fila $M / M / m$ no estado estacionário, com taxa de chegada $\lambda$, pode-se provar que os tempos de saída no sistema também são exponenciais com taxa $\lambda$, ou seja, a taxa de saída é igual 
à taxa de entrada. Portanto, a taxa de produção define a quantidade de clientes que aguardam o atendimento, então tem-se que:

$$
\hat{\lambda}=\lambda_{p}
$$

Logo a taxa de abandono do sistema pode ser calculada como:

$$
\lambda_{\text {abandono }}=\lambda-\lambda_{p}
$$

De posse da taxa de chegada efetiva no sistema é possível calcular várias medidas de desempenho, como: tempo total de espera, tempo de espera na fila, número médio de clientes no sistema, etc.

\section{RESULTADOS}

Com objetivo de encontrar a probabilidade do sistema estar ocioso, a taxa de produção e o tempo médio de sistema, foram realizadas simulações com parâmetros $\lambda=$ $2, \mu=1, l=4, n=6, m=3, p=0,1, \gamma_{1}=2$ e $\gamma_{2}=1$ para uma fila $M / M / 3$. Em que $\lambda$ é a taxa de chegada dos clientes, $\mu$ é a taxa de serviço, $l$ é a capacidade máxima da fila dos clientes do tipo $1, n$ é a capacidade máxima da fila dos clientes do tipo $2, m$ é o número de servidores, $p$ é a probabilidade de chegar um determinando tipo de cliente, $\gamma_{1}$ e $\gamma_{2}$ são as taxas de abandonos dos clientes tipo 1 e tipo 2 respectivamente (SOUZA, 2019). Os valores dos parâmetros foram retirados do artigo do ROUBOS (2013).

Tabela 1. Síntese dos resultados Cliente - Servidor

\begin{tabular}{|c|c|c|}
\hline Resultados & Método Analítico & Método via Simulação \\
\hline $\begin{array}{c}\text { Probabilidade do } \\
\text { sistema vazio }\end{array}$ & $13,6035 \%$ & $\begin{array}{c}13,5884 \% \mathrm{a} \\
14,1326 \%\end{array}$ \\
\hline $\begin{array}{c}\text { Taxa de } \\
\text { produção }\end{array}$ & 1,774025 & $\begin{array}{c}1,771889 \mathrm{a} \\
1,776238\end{array}$ \\
\hline $\begin{array}{c}\text { Tempo médio } \\
\text { de sistema }\end{array}$ & 1,2527 & 1,2555 \\
\hline $\begin{array}{c}\text { Tempo } \\
\text { Computacional (s) }\end{array}$ & 0,0237 & 4,852544 \\
\hline
\end{tabular}

Como pode ser visto na Tabela 1 , foi analisada a probabilidade do sistema ser encontrado vazio, a taxa de produção e o tempo médio de fila e de sistema. O tempo computacional para realizar ambos os métodos foi satisfatório, sendo simulações com pouco custo em relação ao tempo gasto para realiza-las. Mas o método analítico teve um custo menor, em relação ao tempo computacional, que o método por simulação. Os intervalos de $95 \%$ de confiança para o método via simulação foram obtidos por meio de simulações com durações de 10000 segundos, com tempo de aquecimento de 1000 segundos, em 10 rodadas. Juntamente com os resultados da Tabela 1 foi observado o comportamento do sistema ao variar alguns parâmetros.

No gráfico 6 é possível observar que ao aumentar a taxa de serviço, ou seja, um atendimento mais rápido, a taxa de produção aumenta e diminui o número de abandonos, assim aumentando o nível de serviço.

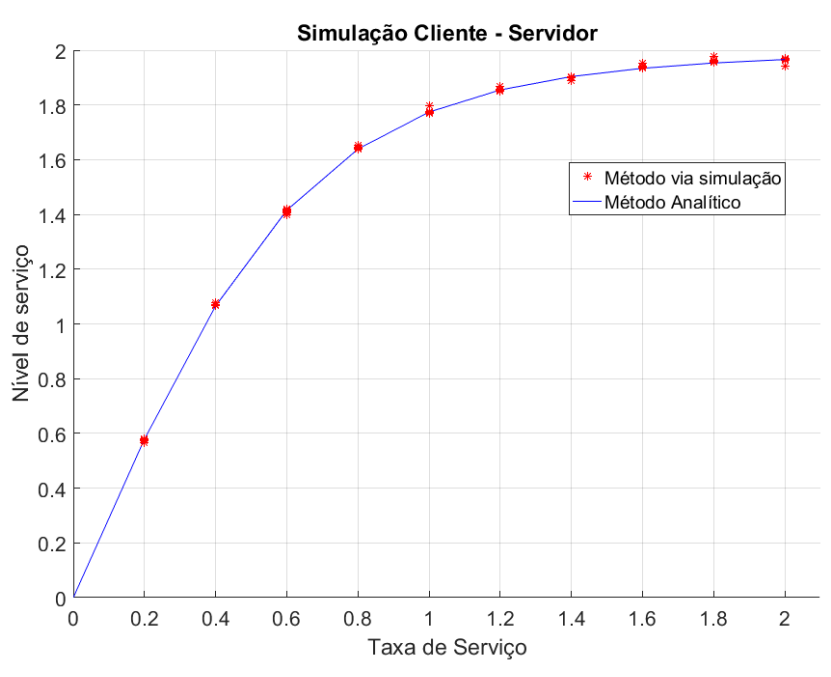

Figura 6. Desempenho em função da taxa de serviço

A Figura 7 mostra o tempo médio de sistema em função do desempenho da taxa de chegada, em que pode-se observar que na medida em que aumenta a taxa de chegada a um aumento no tempo total de sistema.

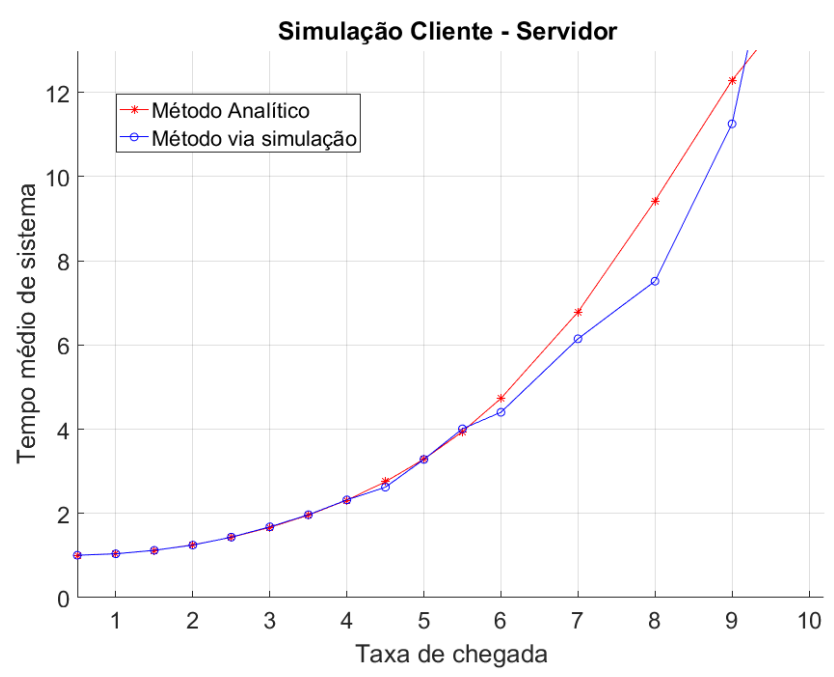

Figura 7. Desempenho do sistema em função da taxa de chegada

Ao alterar a taxa de chegada para análise da eficiência do sistema com três servidores pode-se verificar, através do gráfico da Figura 8, que o nível de serviço, em ambas as metodologias, é satisfatório até uma taxa de chegada de $\lambda=3$, ou seja, tem-se um atendimento adequado até uma chegada de clientes com taxa igual a três, quando a chegada for com uma taxa maior, o número de abandonos começam a crescer e o sistema a ficar menos eficiente.

Outro parâmetro que pode ser observado ao variar a taxa de chegada, nos gráficos da Figura 8, é a taxa de abandono nas duas metodologias, em que pode-se observar um grande aumento nesta taxa devido o cliente permanecer por mais tempo aguardando atendimento, o que gera uma maior taxa média de sistema, ocasionando assim um maior número de abandono. 


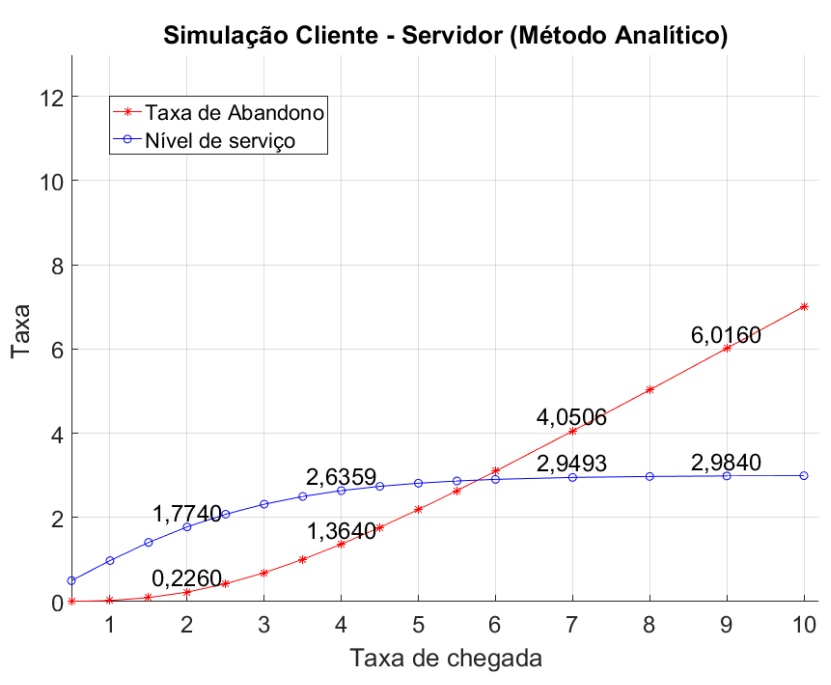

a)Método Analítico

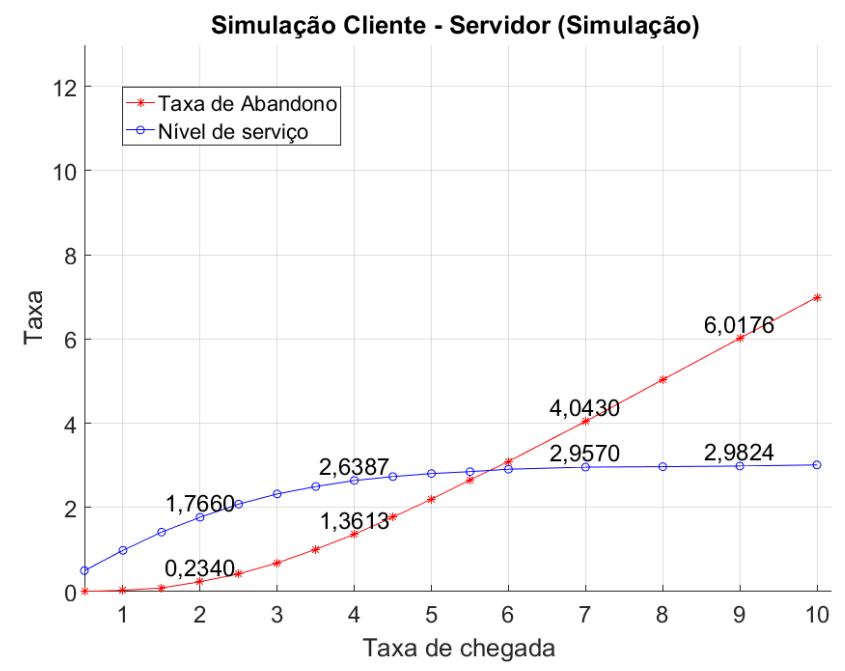

b) Método via simulação

Figura 8. Desempenho em função da taxa de chegada

\section{CONCLUSÃO}

Ao finalizar este trabalho pode-se concluir que foi possível elaborar uma metodologia completa, desde a modelagem até a parte da análise de desempenho dos sistemas, satisfatória em relação ao tempo computacional e os resultados encontrados. Ambos os métodos foram eficientes e com baixo custo computacional para a análise de desempenho dos sistemas de fila cliente - servidor.

Destaca-se a eficiência da modelagem por meio de autômatos estocásticos modulares, facilitando e reduzindo os erros na etapa de modelagem e também possibilitando a simulação computacional e a obtenção da cadeia de Markov. Pode-se enfatizar que ambos os métodos foram eficientes e satisfatórios, mas o método analítico apresenta uma maior eficácia por ter um tempo computacional menor e uma maior quantidade de informações, além de convergir a resultados bem definidos.

Concluindo então que foi possível modelar o comportamento de sistemas de forma modular por meio dos autô- matos estocásticos e, através da biblioteca UltraDES, foi possível realizar a operação da composição paralela para a obtenção da cadeia de Markov em conjunto com as matrizes de transição dos autômatos. Por fim, a análise de desempenho dos parâmetros de interesse foi realizada com sucesso por meio das propriedades dos sistemas juntamente com a teoria de filas.

\section{REFERÊNCIAS}

ABOU-EL-ATA, M.O. ; HARIRI, A. (1992). The $\mathrm{M} / \mathrm{M} / \mathrm{c} / \mathrm{N}$ queue with balking and reneging. Computers e Operations Research, 19, 713 - 716. doi:10.1016/ 0305-0548(92)90010-3.

AL-SEEDY, R.O. ; EL-SHERBINY, A..E.S.S..A.S. (2009). Transient solution of the M/M/c queue with balking and reneging. Computers and Mathematics with Applications, 57, $1280-1285$.

CASSANDRAS, C. G., .L.S. (2008). Introduction to Discrete Event Systems, volume 11. Springer Science Business Media, USA, second edition.

GROSS, D., SHORTLE, J.F., THOMPSON, J.M., and HARRIS, C.M. (2008). Fundamentals of Queueing Theory. Wiley Series in Probability and Statistics. Wiley-Interscience, 4 edition.

HAYES, B. (2013). First links in the Markov chain. 101(2), 92. American Scientist.

LACSED - UFMG (2016). UltraDES, desenvolvido pelo Laboratório de Análise e Controle de Sistemas a Eventos Discretos. www.github.com/lacsed/UltraDES. (Acecsso em: 20 de fevereiro de 2018).

RIBEIRO, R.G. (2018). Performance Evaluation of Stochastic DES Through Analytical Models and Simulation: An Open-Pit Mine Study. Ph.D. thesis, Universidade Federal de Minas Gerais (UFMG).

ROUBOS, A. ; JOUINI, O. (2013). Call centers with hyperexponential patience modeling. 141, $307-315$. doi:10.1016/j.ijpe.2012.08.011.

SOUZA, L. M. G. ; MAIA, C.A. (2018). Análise de desempenho de Sistemas a Eventos Discretos via autômatos estocásticos modulares. 1 - 8. XXII Congresso Brasileiro de Automática.

SOUZA, L.M.G. (2019). Análise de Desempenho de SED via Auiômatos Modulares Estocásticos: Abordagem via simulação computacional e método analítico. Master's thesis, Universidade Federal de Minas Gerais (UFMG).

Wang, K., Li, N., and Jiang, Z. (2010). Queueing system with impatient customers: A review. $82-87$. doi: 10.1109/SOLI.2010.5551611.

WANG, Z. ; LI, S. (2011). Some application of Markov chain to market occupation rate and promotion strategy. 467 - 470. doi:10.1109/ICIC.2011.109. IEEE Fourth International Conference on Information and Computing.

WHITT, W. (2004). Engineering solution of a basic callcenter model. Department of Industrial Engineering and Operations Research - Columbia University, New York.

YUE, D. ; YUE W. ; ZHAO, G. (2014). Analysis of an $\mathrm{M} / \mathrm{M} / \mathrm{c}$ queueing system with impatient customers and synchronous vacations. Journal of Applied Mathematics, 2014, 1 - 12. doi:10.1155/2014/893094. Department of Intelligence and Informatics, Konan University. 